\title{
Evaluation of the shear bond strength of zirconia to a self-adhesive resin cement after different surface treatment
}

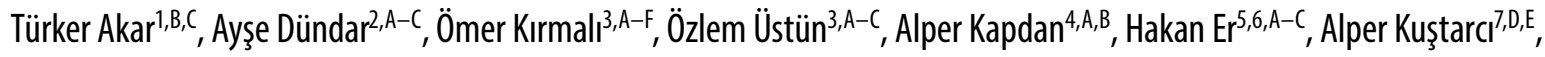 \\ Kürşat Er, ${ }^{7,8, A-F}$, Burak YIImaz ${ }^{9,10, C, D}$ \\ ${ }^{1}$ Department of Prosthodontics, Faculty of Dentistry, Erzincan University, Turkey \\ ${ }^{2}$ Department of Restorative Dentistry, Faculty of Dentistry, Akdeniz University, Antalya, Turkey \\ ${ }^{3}$ Department of Prosthodontics, Faculty of Dentistry, Akdeniz University, Antalya, Turkey \\ ${ }^{4}$ Department of Restorative Dentistry, Faculty of Dentistry, Cumhuriyet University, Sivas, Turkey \\ ${ }^{5}$ Department of Biophysics, Faculty of Medicine, Akdeniz University, Antalya, Turkey \\ ${ }^{6}$ Electron Microscopy Image Analyzing Unit, Faculty of Medicine, Akdeniz University, Antalya, Turkey \\ ${ }^{7}$ Department of Endodontics, Faculty of Dentistry, Akdeniz University, Antalya, Turkey \\ ${ }^{8}$ Department of Endodontics, Faculty of Dentistry, Alanya Alaaddin Keykubat University, Turkey \\ ${ }^{9}$ Department of Reconstructive Dentistry and Gerodontology, School of Dental Medicine, University of Bern, Switzerland \\ ${ }^{10}$ Department of Restorative, Preventive and Pediatric Dentistry, School of Dental Medicine, University of Bern, Switzerland \\ A - research concept and design; $\mathrm{B}$ - collection and/or assembly of data; $\mathrm{C}$ - data analysis and interpretation; \\ $D$ - writing the article; $E$ - critical revision of the article; $F$ - final approval of the article
}

\section{Address for correspondence \\ Kürşat Er \\ E-mail: qursater@hotmail.com}

\section{Funding sources}

The work was supported by the Scientific Research Projects Coordination Unit of Akdeniz University, Antalya, Turkey (grant No. TSA-2015-739).

Conflict of interest None declared

Received on 0ctober 12, 2020 Reviewed on March 30, 2021 Accepted on April 9, 2021

Published online on December 31, 2021

Cite as

Akar T, Dündar A, Krrmalı̈ 0 , et al. Evaluation of the shear bond strength of zirconia to a self-adhesive resin cement after different surface treatment. Dent Med Probl. 2021;58(4):463-472. doi:10.17219/dmp/135652

DOI

$10.17219 / \mathrm{dmp} / 135652$

Copyright

○) 2021 by Wroclaw Medical University

This is an article distributed under the terms of the

Creative Commons Attribution 3.0 Unported License (CC BY 3.0)

(https://creativecommons.org/licenses/by/3.0/).

\begin{abstract}
Background. Bond stability between zirconia and a self-adhesive resin cement is a major concern, and only limited evidence about its longevity is currently available. Moreover, no study has yet comprehensively evaluated the influence of different power levels of the neodymium-doped yttrium-aluminum-garnet (Nd:YAG) laser irradiation on the shear bond strength (SBS) of zirconia to a self-adhesive resin cement.

Objectives. The aim of this study was to evaluate the SBS of pre-sintered and sintered zirconia to a selfadhesive resin cement after various treatment (air abrasion and the Nd:YAG laser irradiation at varying power levels $-1 \mathrm{~W}, 2 \mathrm{~W}$ and $3 \mathrm{~W}$ ).

Material and methods. Ninety-nine zirconia specimens were prepared and divided into 3 groups: control (with no surface treatment); and pre-sintered and sintered groups with surface treatment. Surface treatment was applied before sintering in the pre-sintered group and after sintering in the sintered group. After following all protocols, a resin cement was layered on the zirconia surface. Shear bond strength was measured using a universal testing machine. The results were subjected to the statistical analysis. The surface topography and phase transformation of zirconia were evaluated using the atomic force microscopy (AFM), scanning electron microscopy (SEM) and X-ray diffraction (XRD) analyses after surface treatment.
\end{abstract}

Results. The laser irradiation ( $3 \mathrm{~W}, 1 \mathrm{~W}$ and $2 \mathrm{~W}$ ) of the pre-sintered zirconia surface resulted in the highest SBS values $(p<0.001)$, while the lowest SBS values were obtained with airborne particle abrasion of the pre-sintered and sintered zirconia surfaces.

Conclusions. Laser irradiation increased the SBS of pre-sintered zirconia to a resin cement. Surface treatment with air abrasion had a lesser effect on the SBS values.

Keywords: shear bond strength, zirconia, resin cement, surface treatment, Nd:YAG laser 


\section{Introduction}

Zirconia, especially an yttrium-stabilized tetragonal zirconia polycrystalline (Y-TZP) ceramic, is a durable and commonly used dental material that was introduced for single crowns, ${ }^{1}$ frameworks for fixed partial dentures ${ }^{1,2}$ and implant abutments ${ }^{3}$ with the development of the computer-aided design/computer-aided manufacturing (CAD-CAM) technology. It can be used both in the anterior and posterior regions due to its favorable mechanical properties (high fracture toughness (7-10 MPa) and high flexural strength (700-1,200 MPa)) and color. ${ }^{1-3}$ Zirconia can be used in a monolithic form or can be veneered using feldspathic porcelain.

Zirconia is a polymorphic material. When stimulated, it responds through a phase transformation mechanism, changing from its tetragonal $(\mathrm{t})$ to monoclinic $(\mathrm{m})$ phase, which results in superficial compressive stress with a grain volume increase of 3-5\%. This prevents the further propagation of cracks and increases the toughness of zirconia. ${ }^{4}$

With reliable chemical bonding between a resin cement and a restorative material, more tooth structures can be preserved and more durable restorations with short clinical crowns can be performed..$^{5}$ A strong bond depends on many factors, such as surface roughness, wettability of the resin cement, and bonding ability of the cement and its chemical ingredients in particular. ${ }^{6}$ It has been stated that the application of the 10-methacryloyloxydecyl dihydrogen phosphate (10-MDP) monomer containing a bonding agent is the key factor for a durable bond between zirconia and the tooth, and that the bonding agent improves the wettability of the treated zirconia surface. ${ }^{7}$ Several studies have been conducted on the effect of different zirconia surface treatment on the quality of the resin cement-zirconia bond. ${ }^{8-10}$ Common treatment options include the application of mineral acids, such as hydrofluoric acid and phosphoric acid, selective infiltration etching, experimental treatment with a hot-etching solution, and plasma spray treatment. ${ }^{9,11,12}$ Hydrofluoric acid has the ability to remove the glass phase of the ceramic structure in a process that produces a micro-retentive surface with high free energy, which increases the adhesion between the resin cement and the ceramic through the application of a silane agent. However, acid etching is not suitable for zirconia, as it does not have a glassy phase.

Various mechanical treatment techniques, including air abrasion with $\mathrm{Al}_{2} \mathrm{O}_{3}$ particles of the preferred size range of 25-250 $\mu \mathrm{m}$ and laser surface treatment, such as irradiation with the neodymium-doped yttrium-aluminum-garnet (Nd:YAG), erbium-doped yttrium-aluminum-garnet (Er:YAG), erbium, chromium-doped yttrium-scandiumgallium-garnet (Er,Cr:YSGG), and carbon dioxide $\left(\mathrm{CO}_{2}\right)$ lasers, have also been applied and evaluated for improving the resin cement-zirconia adhesion. ${ }^{6-9}$

Air abrasion with $\mathrm{Al}_{2} \mathrm{O}_{3}$ particles combined with a 10-MDP-containing primer/resin cement has been suggested as an effective technique for bonding. ${ }^{7}$ However, it has been criticized for causing micro-cracks within zirconia by increasing the content of the $m$ phase, ${ }^{6,9,12}$ and additional heat treatment would be required to decrease the $\mathrm{m}$ phase. ${ }^{10}$ As an alternative, some researchers proposed different kinds of pretreatment of the pre-sintered zirconia surface ${ }^{5,10,13}$ and found that the value of the zirconia shear bond strength (SBS) significantly increased after the air abrasion treatment. ${ }^{5}$ Additionally, the air abrasion of pre-sintered zirconia followed by sintering could provide some advantages, such as a higher surface roughness with deep crevices and projections, which increases the content of the $t$ phase, and no additional heat treatment but air abrasion is needed after the sintering procedure. ${ }^{10}$ Although the air abrasion technique remains questionable, it is still regarded as effective.

Meanwhile, various lasers have been applied to achieve increased surface roughness, wettability and SBS of zirconia in its sintered form. It has been reported that the Nd:YAG, Er:YAG and Er,Cr:YSGG lasers could help to produce a strong bond at the resin cement-zirconia interface. ${ }^{4,14}$ Among the 3 effective lasers (Nd:YAG, Er:YAG and Er,Cr:YSGG), the highest surface roughness of zirconia has been recorded with the use of the Nd:YAG laser. ${ }^{9}$ Gandolfi Paranhos et al. reported that they achieved a higher surface roughness of zirconia with the Nd:YAG laser as compared to the $\mathrm{CO}_{2}$ laser. ${ }^{15}$ However, some researchers reported that lasers might induce phase transformation through a high laser power and uncontrolled temperature, which may also damage the zirconia surface. ${ }^{9,16,17}$ The use of laser irradiation for the treatment of the pre-sintered zirconia surface is an alternative to the treatment of sintered zirconia, and it also helps to improve SBS at the resin cement-zirconia interface. ${ }^{13,14}$ It has been previously reported that prolonging the irradiation time in addition to increasing the irradiation power does not increase the adhesion strength of ceramics and causes defects in the material. ${ }^{18}$ For this reason, the questions how much irradiation power is used and for how long the zirconia material is irradiated are important in terms of SBS of the material and defects that may occur in it. In this study, the Nd:YAG laser was used, as it increases the surface roughness and wettability of zirconia, and thus a high value of SBS can be achieved.

Several kinds of surface treatment of pre-sintered or sintered zirconia have been recommended to improve the SBS of zirconia to a resin cement. However, the effect of laser treatment at varying power levels on the SBS of zirconia in different forms to a resin cement is not well known. The aim of this in vitro study was to evaluate the effect of different surface treatment (airborne particle abrasion and the Nd:YAG laser irradiation at varying power levels) on the SBS of pre-sintered or sintered zirconia to a resin cement. The null hypothesis was that the zirconia form and surface treatment would not affect the resin cementzirconia SBS values. Also, the surface topography and phase transformation of zirconia were evaluated using the atomic force microscopy (AFM), scanning electron microscopy (SEM) and X-ray diffraction (XRD) analyses. 


\section{Material and methods}

\section{Sample preparation}

Ninety-nine pre-sintered zirconia specimens (Kuraray Noritake Dental Inc., Nagoya, Japan), $7 \mathrm{~mm}$ in diameter and $3 \mathrm{~mm}$ in height, were prepared using a CAD-CAM milling device (Yenamak D50; Yenadent, Istanbul, Turkey). Of those, 9 specimens were used for the AFM, SEM and XRD analyses. A grinding machine (Phoenix Beta Grinder/Polisher; Buehler, Esslingen am Neckar, Germany) was used with 600-, 800- and 1,200-grit silicon carbide abrasives (English Abrasives \& Chemicals Ltd., Stafford, $\mathrm{UK})$ for $15 \mathrm{~s}$ to form a standard bonding surface on the zirconia specimens. A flowchart of the test protocol used in the study is illustrated in Fig. 1.

Forty specimens were sintered at $1,500^{\circ} \mathrm{C}$ for $8 \mathrm{~h}$, according to the manufacturer's recommendations (the sintered group). The specimens were divided into 3 groups: pre-sintered control (pre-sintered-C); pre-sintered; and sintered. The pre-sintered and sintered groups were then divided into 4 subgroups according to the surface treatment technique. Surface treatment and bonding procedures were performed by the same operator. The control group specimens received no further surface pretreatment. The airborne particle abrasion subgroups consisted of pre-sintered and sintered zirconia specimens that were abraded with 120 -micrometer $\mathrm{Al}_{2} \mathrm{O}_{3}$ particles at a pressure of $200 \mathrm{kPa}$ for $20 \mathrm{~s}$ at a distance of $10 \mathrm{~mm}$. The laser

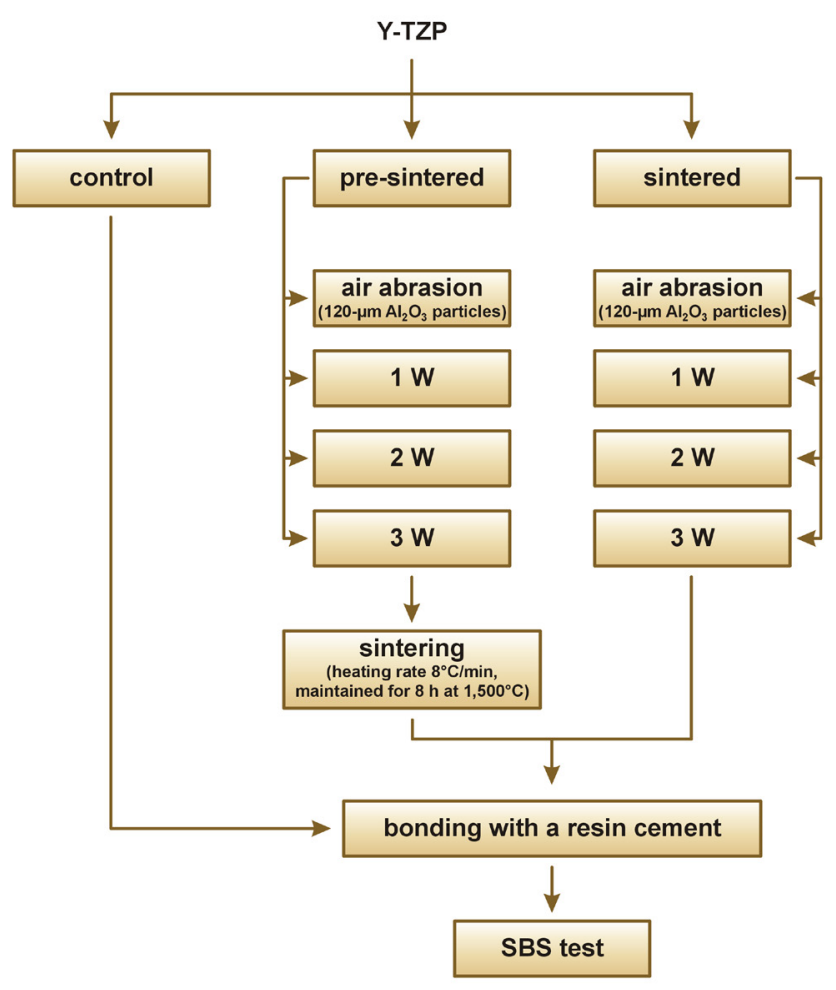

Fig. 1. Flowchart of the test protocol used in the study Y-TZP - yttrium-stabilized tetragonal zirconia polycrystalline; SBS - shear bond strength. subgroups contained specimens that were irradiated with the Smarty A10 Nd:YAG laser (DEKA Laser, Calenzano, Italy), which produces laser pulses at a wavelength of $1.06 \mu \mathrm{m}$. The optical fiber of the laser was $300 \mu \mathrm{m}$ in diameter and was held manually perpendicular to the zirconia ceramic for $20 \mathrm{~s}$ at a distance of $1 \mathrm{~mm}$, as described in previous studies. ${ }^{7,8}$ The laser parameters included a pulse energy of $100 \mathrm{~mJ}$, a repetition rate of 10,20 and $30 \mathrm{~Hz}$, and a pulse duration of $320 \mu$ s (Table 1 ).

Table 1. Laser parameters used in the study

\begin{tabular}{|lc|}
\multicolumn{1}{|c}{ Parameter } & Nd:YAG laser characteristics \\
\hline Manufacturer & DEKA Laser, Calenzano, Italy \\
Model identifier & Smarty A10 \\
Wavelength & $1,064 \mathrm{~nm}$ \\
Delivery system & 300-micrometer quartz optical fiber \\
Power & $1 \mathrm{~W}, 2 \mathrm{~W}, 3 \mathrm{~W}$ \\
Power density & $1.4 \mathrm{~W} / \mathrm{cm}^{2}$ \\
Energy density & $85 \mathrm{~J} / \mathrm{cm}^{2}$ \\
Duration & $20 \mathrm{~s}$ at a distance of $1 \mathrm{~mm}$ \\
Repetition rate & $10 \mathrm{~Hz}, 20 \mathrm{~Hz}, 30 \mathrm{~Hz}$ \\
Pulse duration & $320 \mu \mathrm{ss}$ \\
\hline
\end{tabular}

Nd:YAG - neodymium-doped yttrium-aluminum-garnet.

\section{Shear bond strength test}

All pre-sintered specimens were sintered at $1,500^{\circ} \mathrm{C}$ for $8 \mathrm{~h}$ again. A self-adhesive dual-polymerizing resin cement (Bifix SE, Lot: 1824158; VOCO, Cuxhaven, Germany) was applied to both the untreated and treated bonding surfaces with the use of cylindrical Tygon ${ }^{\circledR}$ tubes, $3 \mathrm{~mm}$ in diameter and $3 \mathrm{~mm}$ in height, in accordance with the manufacturer's recommendations. The Tygon tubes were then removed and all resin cement-zirconia specimens were immersed in distilled water at $37^{\circ} \mathrm{C}$ for $72 \mathrm{~h}$ before testing SBS. Using a crosshead speed of $1 \mathrm{~mm} / \mathrm{min}$, each specimen was loaded to fracture in a universal testing machine (Lloyd LF Plus; AMETEK, Meerbusch, Germany) for the SBS test.

\section{AFM analysis}

After surface treatment, 1 randomly selected specimen from each group was studied by means of an AFM (Park Systems, Suwon, South Korea). The AFM was used in the non-contact mode with a cantilever (NSC 36; MicroMasch, Tallinn, Estonia) with an aluminum-doped silicon tip (a radius of curvature of $10 \mathrm{~nm}$ or less). The measurements were performed with the aid of a computer program (XEP software; Park Systems). The grains were measured over an area of $1 \times 1 \mu \mathrm{m}$. All images of $512 \times 512 \mathrm{px}$ were obtained with a low scanning frequency $(0.3 \mathrm{~Hz})$ from each surface. 


\section{SEM analysis and the fracture pattern analysis}

One specimen was selected from each group and analyzed with an SEM (JSM-6060LV; JEOL Ltd., Tokyo, Japan) at $\times 500$ and $\times 1,000$ magnification after being coated with gold-palladium. After the SBS test, the fracture patterns were detected using a stereomicroscope (Stemi ${ }^{\text {TM }}$ DV4; Carl Zeiss Microscopy, Göttingen, Germany) at $\times 32$ magnification. The fracture patterns were classified as adhesive failure (at the interface between the zirconia surface and the cement), cohesive failure (within the cement) or mixed failure (adhesive and cohesive).

\section{XRD analysis}

The XRD patterns of 1 sample from each group were obtained with an X-ray diffractometer (Empyrean; Malvern Panalytical Ltd., Malvern, UK) using $\mathrm{Cu} \mathrm{K} \alpha$ radiation $(\lambda=1.5406 \AA ; 45 \mathrm{kV}$ and $40 \mathrm{~mA})$. The calculations were based on the Garvie and Nicholson method, ${ }^{19}$ which is a currently accepted one.

\section{Statistical analysis}

In order to analyze differences in the SBS values between the groups, the one-way Welch $F$ test (without assessing the homogeneity of variance) and the Games-Howell post-hoc test were used. The statistical analysis was performed using the IBM SPSS Statistics for Windows software, v. 22.0 (IBM Corp., Armonk, USA), with a significance level at $p<0.05$.

\section{Results}

Shear bond strength was significantly affected by the zirconia form (pre-sintered or sintered) and by surface treatment (Table 2). The SBS values for each group with different surface treatment are displayed in Table 3. The SBS values obtained with laser irradiation at power levels of $1 \mathrm{~W}, 2 \mathrm{~W}$ and $3 \mathrm{~W}$ on the pre-sintered zirconia surface were significantly higher as compared to the untreated surface and the sintered subgroups. Laser irradiation at varying power levels slightly increased the SBS values of the sintered zirconia surface with regard to the air abrasion sintered subgroup except for the $3 \mathrm{~W}$ subgroup. However, there were no significant differences with regard to the control group. The laser irradiation pre-sintered subgroups showed the highest $\mathrm{SBS}$ values $(18.3 \pm 2.7 \mathrm{MPa}$ $(3 \mathrm{~W}), 14.1 \pm 2.8 \mathrm{MPa}(1 \mathrm{~W})$ and $13.1 \pm 2.7 \mathrm{MPa}(2 \mathrm{~W}))$ $(p<0.001)$. The lowest SBS values were obtained with airborne particle abrasion in the case of both pre-sintered and sintered forms.

The analysis of failure revealed that fracturing occurred predominantly at the resin cement-zirconia interface
Table 2. Results of the one-way Welch $F$ test with regard to the shear bond strength (SBS) values [MPa] in all groups

\begin{tabular}{|c|c|c|c|c|}
\hline $\begin{array}{c}\text { Statistical } \\
\text { test }\end{array}$ & df1 & df2 & $F$ & $p$-value \\
\hline Welch F test & 8 & 33.119 & $42.610^{\text {a }}$ & $0.000^{*}$ \\
\hline
\end{tabular}

df - degree of freedom; ${ }^{\text {a }}$ asymptotically F-distributed; * statistically significant.

Table 3. Shear bond strength (SBS) values for all groups $(n=11)$

\begin{tabular}{l|c|}
\multicolumn{1}{c|}{ Groups } & \multicolumn{1}{c|}{$\begin{array}{c}\text { SBS } \\
{[\mathrm{MPa}]}\end{array}$} \\
\hline Control & $6.0 \pm 1.1^{\mathrm{a}, \mathrm{c}}$ \\
Air abrasion pre-sintered & $4.5 \pm 1.1^{\mathrm{a}, \mathrm{d}}$ \\
Air abrasion sintered & $4.7 \pm 0.9^{\mathrm{a}}$ \\
1 W laser irradiation pre-sintered & $14.1 \pm 2.8^{\mathrm{b}, \mathrm{e}}$ \\
1 W laser irradiation sintered & $6.8 \pm 0.5^{\mathrm{c}}$ \\
2W laser irradiation pre-sintered & $13.1 \pm 2.7^{\mathrm{b}}$ \\
2W laser irradiation sintered & $6.1 \pm 0.7^{\mathrm{c}, \mathrm{d}}$ \\
3W laser irradiation pre-sintered & $18.3 \pm 2.7^{\mathrm{e}}$ \\
3 W laser irradiation sintered & $6.0 \pm 1.3^{\mathrm{a}, \mathrm{c}}$ \\
\hline
\end{tabular}

Data presented as mean \pm standard deviation $(M \pm S D)$. The same letters in superscript show no statistically significant difference $(p>0.05)$.

(48.88\%). Cohesive failure in the resin cement was infrequent (20.00\%) and mixed failure (adhesive and cohesive) occurred with a moderate frequency (31.12\%).

The relative amount of monoclinic zirconia which was defined with the XRD analysis on the untreated and treated surfaces (pre-sintered and sintered) of all specimens are presented in Fig. 2. Nearly 100\% t phase was obtained on pre-sintered and sintered zirconia before surface treatment. After mechanical treatment (airborne particle abrasion or laser irradiation), there was an increase in the $\mathrm{m}$ content of zirconia, especially in the pre-sintered subgroups. After the sintering process was performed in the pre-sintered subgroups, the m peaks disappeared and the $\mathrm{m}$ content was similar to that of the untreated surface (control group).

The SEM and AFM analyses revealed that the zirconia surface was modified after different treatment. While there were more homogeneous layers formed on the airborne particle-abraded (pre-sintered and sintered) surfaces, which did not show any major morphological differences as compared to the untreated surface (Fig. 3), the laser-irradiated pre-sintered surfaces were irregular, with melting and the formation of pits (Fig. 4). The laser irradiation of the presintered specimens led to the formation of well-defined micro-sized elevated and depressed areas, which was possibly due to the high impact of the laser power (Fig. 4). Nevertheless, the treatment of the sintered surfaces with laser irradiation resulted in wide carbonization areas with macro-cracks as compared to the control group (Fig. 5).

The areas created on the pre-sintered surfaces by laser irradiation could provide retention for the bonding of the resin cement (Fig. 6). 

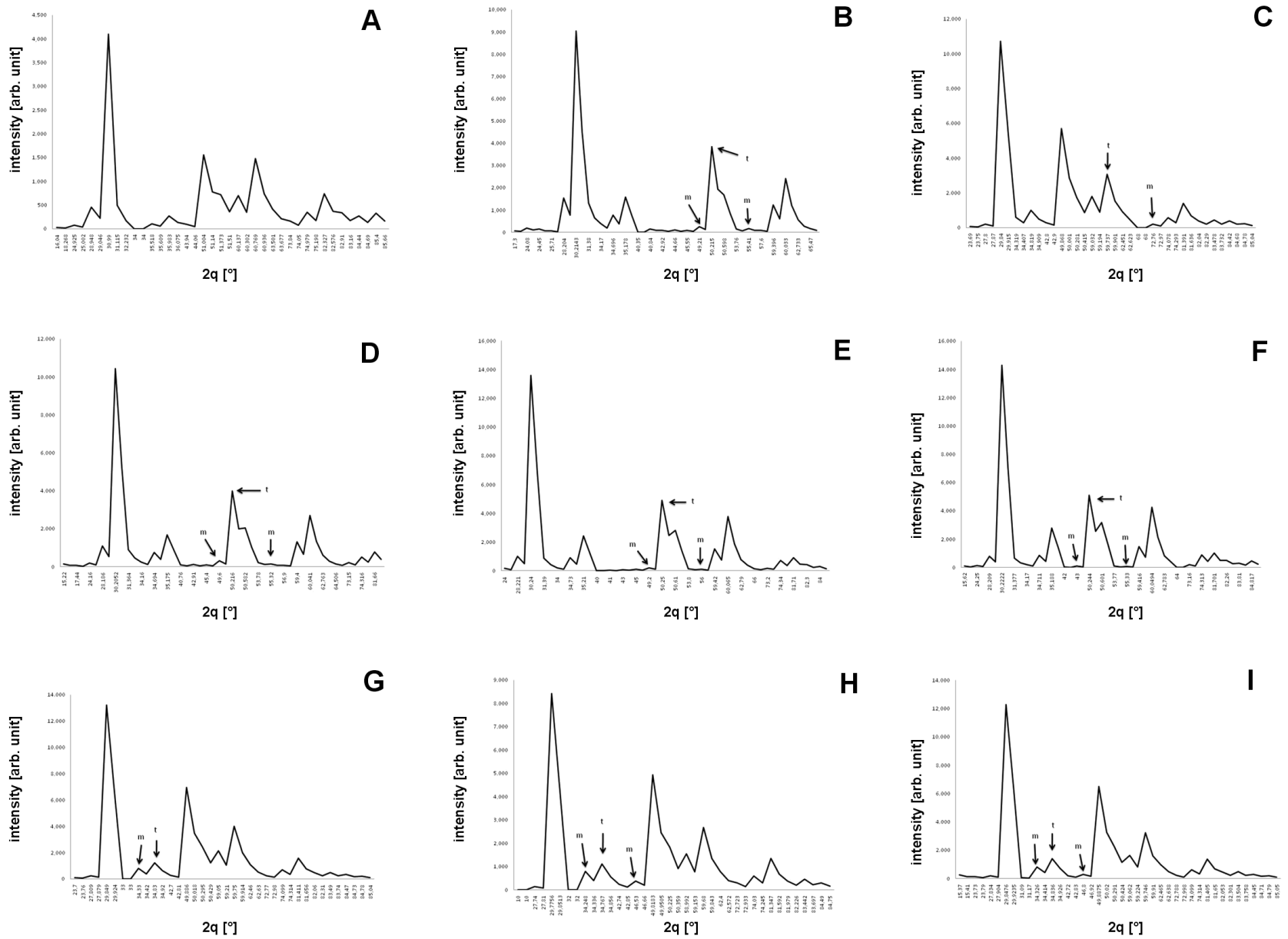

Fig. 2. Results of the X-ray diffraction (XRD) analysis of the pre-sintered and sintered zirconia surfaces

A - untreated pre-sintered; B - air-abraded pre-sintered; C - air-abraded sintered; D - laser-irradiated (1 W) pre-sintered; E - laser-irradiated (2 W) pre-sintered; F - laser-irradiated (3 W) pre-sintered; G - laser-irradiated (1 W) sintered; H - laser-irradiated (2 W) sintered; I - laser-irradiated (3 W) sintered.

Sintered zirconia had a rougher surface than the untreated pre-sintered zirconia (Fig. 3A, 3B). It was observed that the sandblasting of sintered zirconia provided a rougher surface as compared to the sandblasting of presintered zirconia (Fig. 3D-G). The laser irradiation of the pre-sintered and sintered zirconia surfaces resulted in rougher surface textures as compared to the groups without laser irradiation (Fig. 3-5).

\section{Discussion}

The null hypothesis of this study was rejected, since the treatment of pre-sintered zirconia affected the SBS of zirconia to the resin cement. In addition, the mechanical treatment (airborne particle abrasion and laser irradiation at different power levels) of zirconia increased the transformation of the $\mathrm{m}$ phase.

The accurate measurement of bond strength at the zirconia-veneer porcelain interface is quite complex. However, the SBS test is a common method applicable to zirconia-based ceramic systems. ${ }^{20}$ As most of the stresses related to the fracture of the bond between the tooth and the restoration are the stresses of shearing in clinical terms, ${ }^{21}$ and since the applied forces are perpendicular to the bond surface, the small cross-sectional area of the bond surface virtually eliminates the combination of structural defects that significantly affect the test readings. ${ }^{22}$ Therefore, in this study, the SBS test was used to assess the SBS of zirconia to the resin cement. Consequently, no further specimen processing was required after the bonding procedure.

In addition to obtaining a reliable bond between zirconia and a resin cement, the purpose of the zirconia surface treatment is to increase the surface area, which can result in improved wettability. ${ }^{7,8}$ A proportionally larger area has a high surface energy, which can improve mechanical bonding to the zirconia surface. ${ }^{9,18}$ Airborne particle abrasion has gained popularity with regard to obtaining roughness on the zirconia surface, ${ }^{23}$ but conflicting results were reported in some related studies..$^{8,24,25}$ 

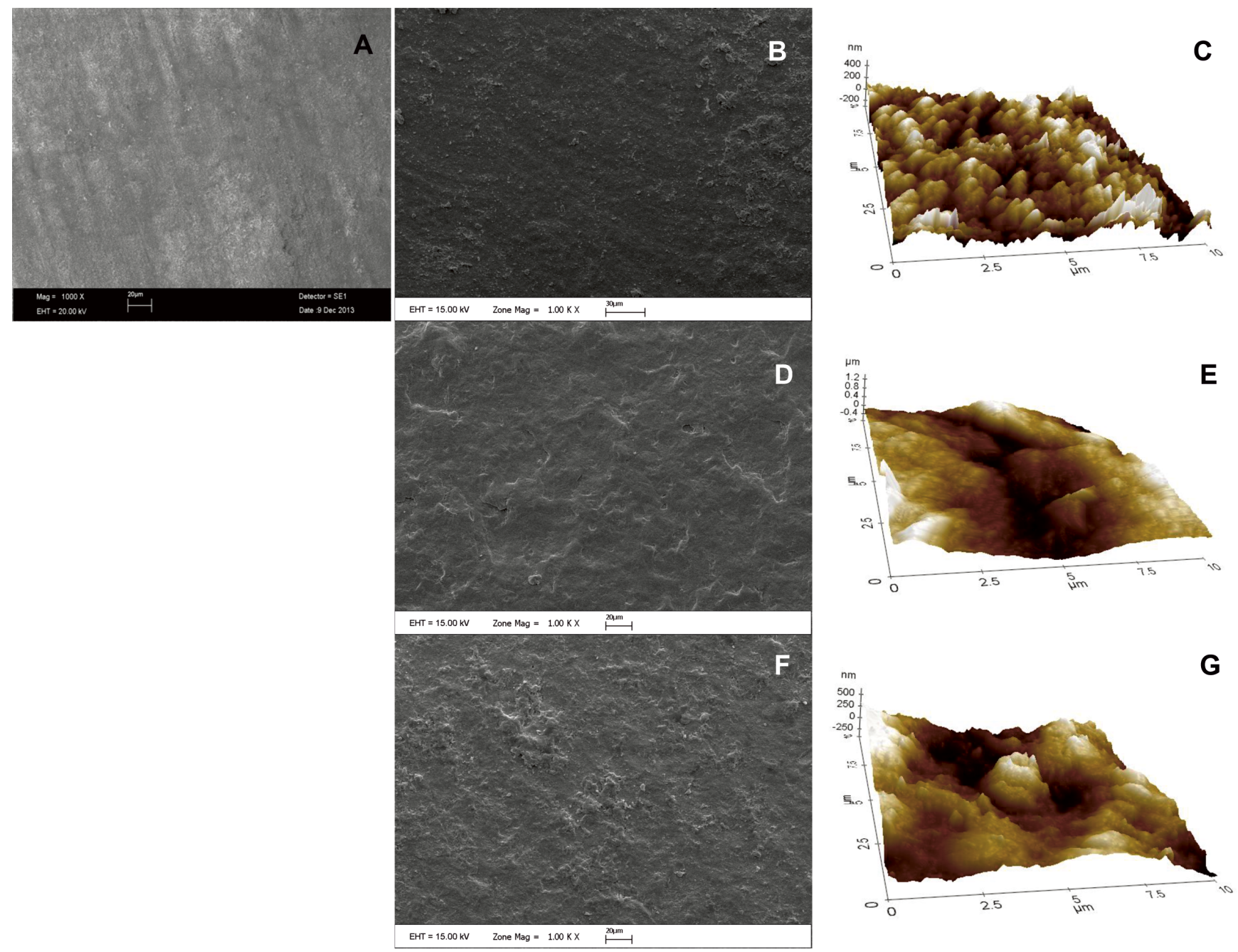

Fig. 3. Scanning electron microscopy (SEM) image of the untreated pre-sintered zirconia surface (A), and the SEM and atomic force microscopy (AFM) images after sintering $(B, C)$, the air abrasion of the pre-sintered zirconia surface $(D, E)$ and the air abrasion of the sintered zirconia surface $(F, G)$

magnification $\times 1,000$.

In many studies applying surface treatment, where SBS and surface roughness were evaluated, there was no correlation between the SBS values and the surface roughness values. ${ }^{17,26}$ A strong bond depends on many factors, such as roughness, wettability of the resin cement, cementation with self-, light-, or dual curing, and composition of the materials. Roughness is just one of them.

The application of sandblasting has the disadvantage of slightly changing the surface chemistry due to the alumina contamination it causes. ${ }^{27}$ The roughening of the zirconia surface with the airborne particle abrasion method has been suggested because of the absence of polycrystalline and glass phases on the zirconia surface, unlike in the case of glass-ceramics. ${ }^{8}$ Some manufacturers recommend the airborne particle abrasion of the zirconia surface before cementation as a routine surface treatment technique. In a previous study, the application of airborne particle abrasion with 50-micrometer $\mathrm{Al}_{2} \mathrm{O}_{3}$ particles to sintered zirconia enhanced the interfacial SBS. ${ }^{14}$ It has been suggested that increasing the irregularities on the airborne particle-abraded zirconia surface may enhance the strength of the bond. ${ }^{14}$ Several studies reported similar results, with a 10-MDP-containing resin cement providing the highest SBS values when used on the zirconia surface treated with airborne particle abrasion with 50-micrometer alumina particles. ${ }^{9,10}$ Contrarily, the SBS values of the airborne particle abrasion group which was treated with 120-micrometer alumina particles were the smallest among all test groups in the current study. This indicates that the airborne particleabraded surface may provide an unsatisfactory resin cement-zirconia bond. This is supported by the SEM and AFM images, which revealed no effect of airborne particle abrasion in modifying the zirconia surface, i.e., the surface was relatively smooth (Fig. 3D-G). Additionally, for the specimens treated with airborne particle abrasion (sintered and pre-sintered), adhesive failure was observed in most of the debonded specimens. Some kinds of mechanical treatment (airborne particle abrasion, laser irradiation, etc.) may damage the zirconia surface by increasing the content of the $\mathrm{m}$ phase. In the current study, it was also found that airborne particle abrasion resulted in the transformation of the $\mathrm{m}$ phase $(6.6 \%)$. Recently, some researchers have 

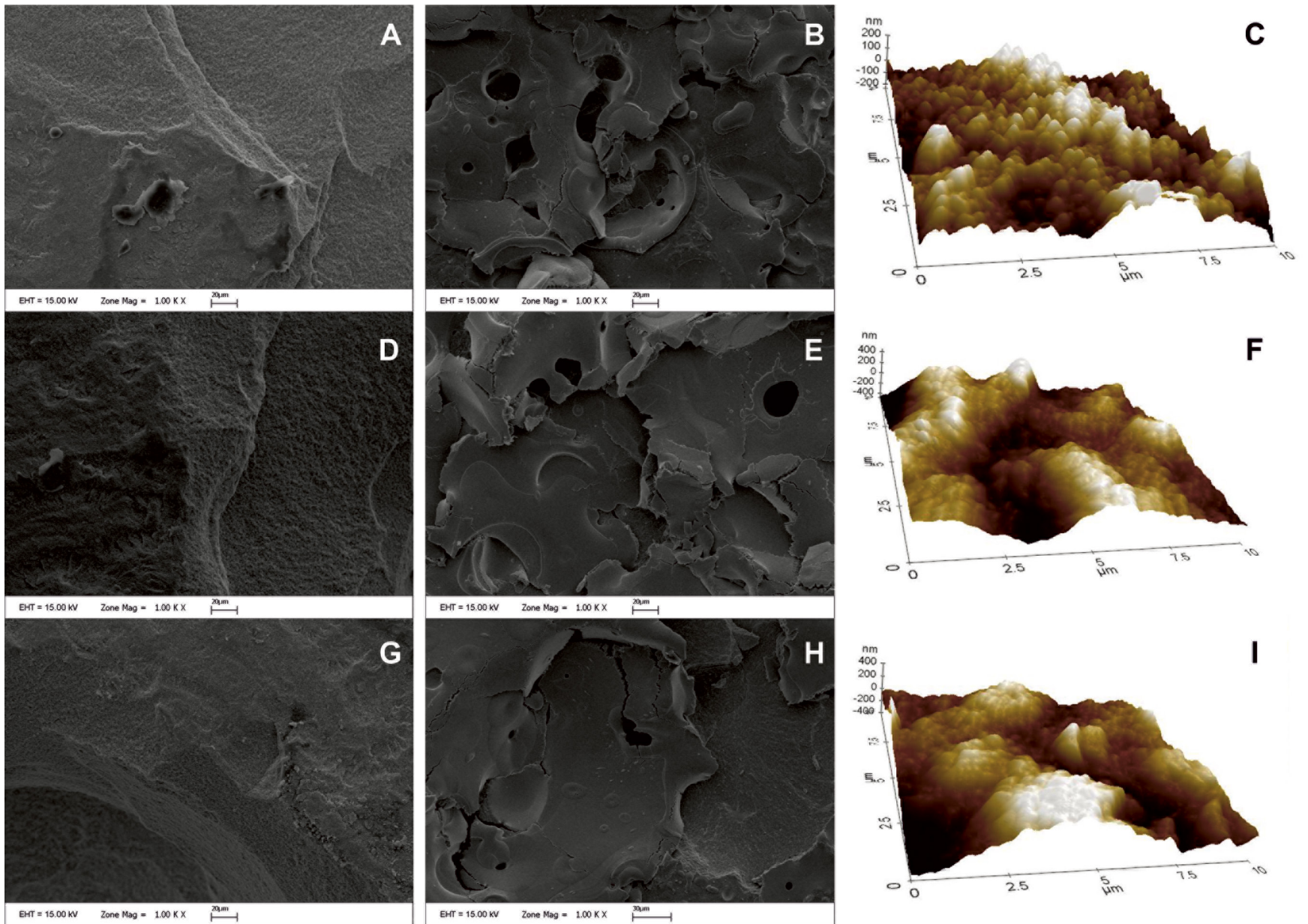

Fig. 4. Scanning electron microscopy (SEM) images of the laser-irradiated pre-sintered zirconia surfaces ( $A-1 \mathrm{~W} ; \mathrm{D}-2 \mathrm{~W} ; \mathrm{G}-3 \mathrm{~W})$, and the SEM and atomic force microscopy (AFM) images after sintering (B, C - $1 \mathrm{~W} ; \mathrm{E}, \mathrm{F}-2 \mathrm{~W} ; \mathrm{H}, \mathrm{I}-3 \mathrm{~W})$ magnification $\times 1,000$

started using the airborne particle abrasion treatment of the pre-sintered zirconia surface to increase retention to zirconia. ${ }^{10,13,14}$ However, it is believed that airborne particle abrasion generates stress on the pre-sintered zirconia surface and increases the content of the $m$ phase. Thus, the values of the $\mathrm{m}$ phase dropped to almost zero after sintering in the present study. This might be explained by the regeneration firing procedure, which induced the $\mathrm{m}-\mathrm{t}$ phase transformation. In addition, in the laser irradiation 1-3 W pre-sintered subgroups (Fig. 2D-F), XRD patterns revealed a lower $\mathrm{m}$ phase than in other laser irradiation subgroups. The $\mathrm{m}-\mathrm{t}$ phase transformation occurred during the sintering process and the final proportion of the $\mathrm{m}$ phase was almost zero in the pre-sintered laser irradiation subgroups, but $38.8 \%$, $51.5 \%$ and $44.7 \%$ in the laser irradiation sintered subgroups, respectively for $1 \mathrm{~W}, 2 \mathrm{~W}$ and $3 \mathrm{~W}$.

To date, numerous studies have been published about the effect of laser treatment on the SBS of zirconia to a resin cement or a veneer ceramic as well as on the roughness of zirconia. ${ }^{8-10,14,17}$ Laser etching is a simple and effective technique for surface treatment. It removes organic and inorganic tissue particles through a process called ablation of dental tissue and by melting the surface dental materials through the absorption of laser energy. ${ }^{6}$ The present study revealed that the laser irradiation of sintered zirconia did not have a significant effect on the SBS of zirconia to the resin cement when compared to the untreated surface. This could be attributed to a high laser energy that caused the formation of cracks on the zirconia surface due to temperature changes, which can create internal tension during laser irradiation (Fig. 5A, 5C and 5E). Low energy levels had a less destructive effect on the zirconia surface, as the SBS values slightly increased in the $1 \mathrm{~W}$ and $2 \mathrm{~W}$ subgroups, and decreased in the $3 \mathrm{~W}$ subgroup. On the other hand, the results of the current study are in conflict with a previous one, where the Nd:YAG laser irradiation (a pulse duration of $200 \mathrm{~mJ}$ and a repetition rate of $10 \mathrm{~Hz}$ ) enhanced the SBS of the resin cement-zirconia bond. ${ }^{28}$ The researchers also reported that the relative $\mathrm{m}$ phase proportion of laserirradiated zirconia specimens was $26.5 \%$ and $30.5 \%$ for a long and a short pulse duration, respectively. The difference was due to a longer irradiation time (60 s) and the fact that the surface of zirconia was coated with graphite. ${ }^{28}$ Similarly, one study revealed that the Nd:YAG laser could enhance SBS. ${ }^{1}$ In addition, with regard to the failure types at the resin cement-zirconia interface, $50 \%$ referred to mixed failure 

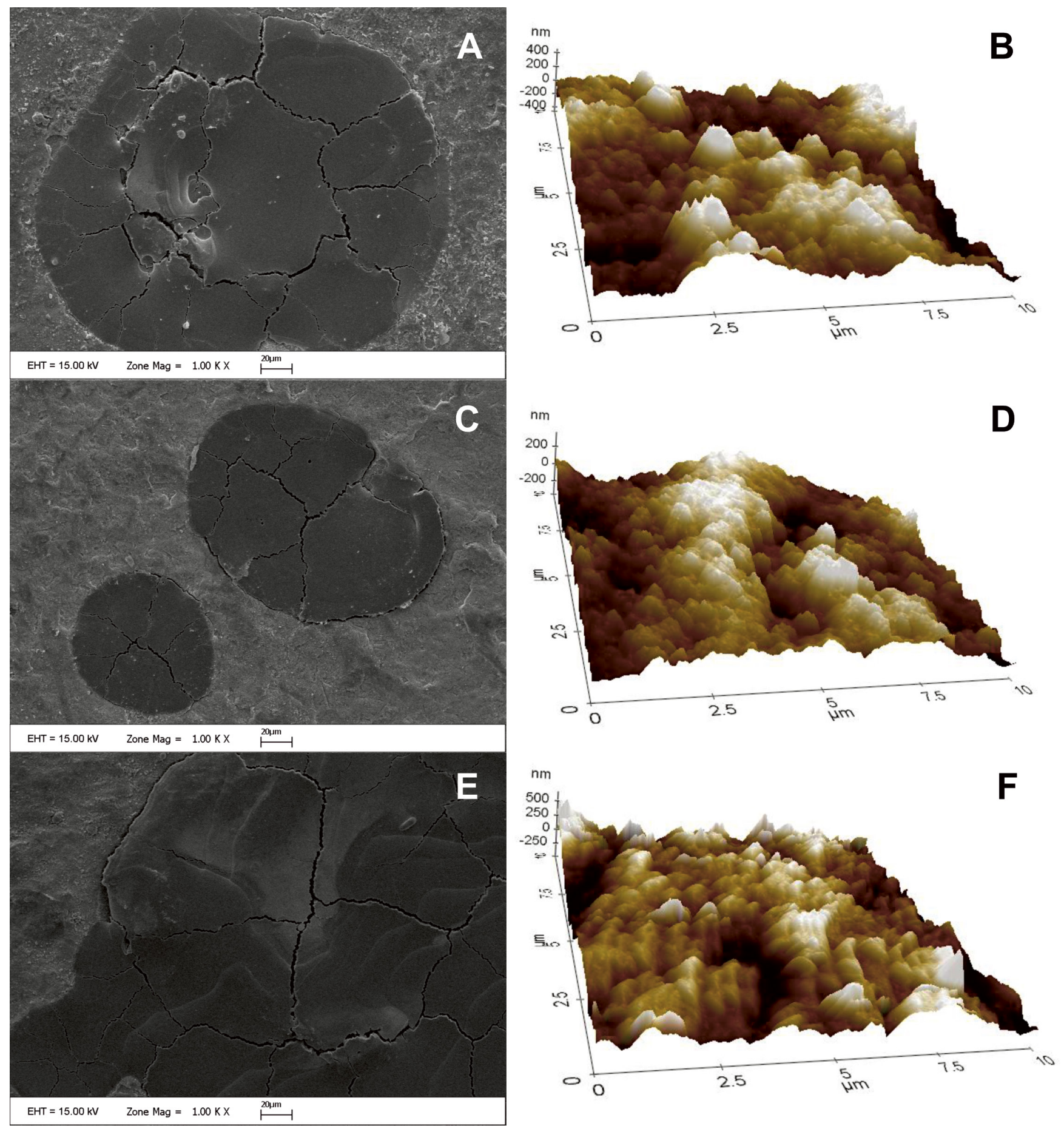

Fig. 5. Scanning electron microscopy (SEM) and atomic force microscopy (AFM) images of the laser-irradiated sintered zirconia surfaces

$(A, B-1 W ; C, D-2 W ; E, F-3 W)$

magnification $\times 1,000$.

in the Nd:YAG laser group. ${ }^{1}$ A previous study showed that a durable resin cement-zirconia bond was achieved after performing the Nd:YAG laser irradiation and using resin cements containing the 10-MDP monomer. ${ }^{15}$ Further studies should be performed to develop new techniques with reliable procedures.

The $\mathrm{m}-\mathrm{t}$ phase transformation enhances the fracture tendency in zirconia. Various kinds of mechanical treatment are responsible for triggering the transformation from the $m$ to the $t$ phase and regeneration firing should be done. ${ }^{10}$ The airborne particle abrasion of pre-sintered zirconia in advance, and then performing the sintering procedure increased the $\mathrm{m}-\mathrm{t}$ phase transformation by creating a retention area on the surface. ${ }^{10}$ The effect of laser irradiation on the pre-sintered zirconia surface has been reported and the values of zirconia SBS have been stated to be significantly increased after irradiating zirconia with various lasers. ${ }^{13,14}$ Such changes in the 

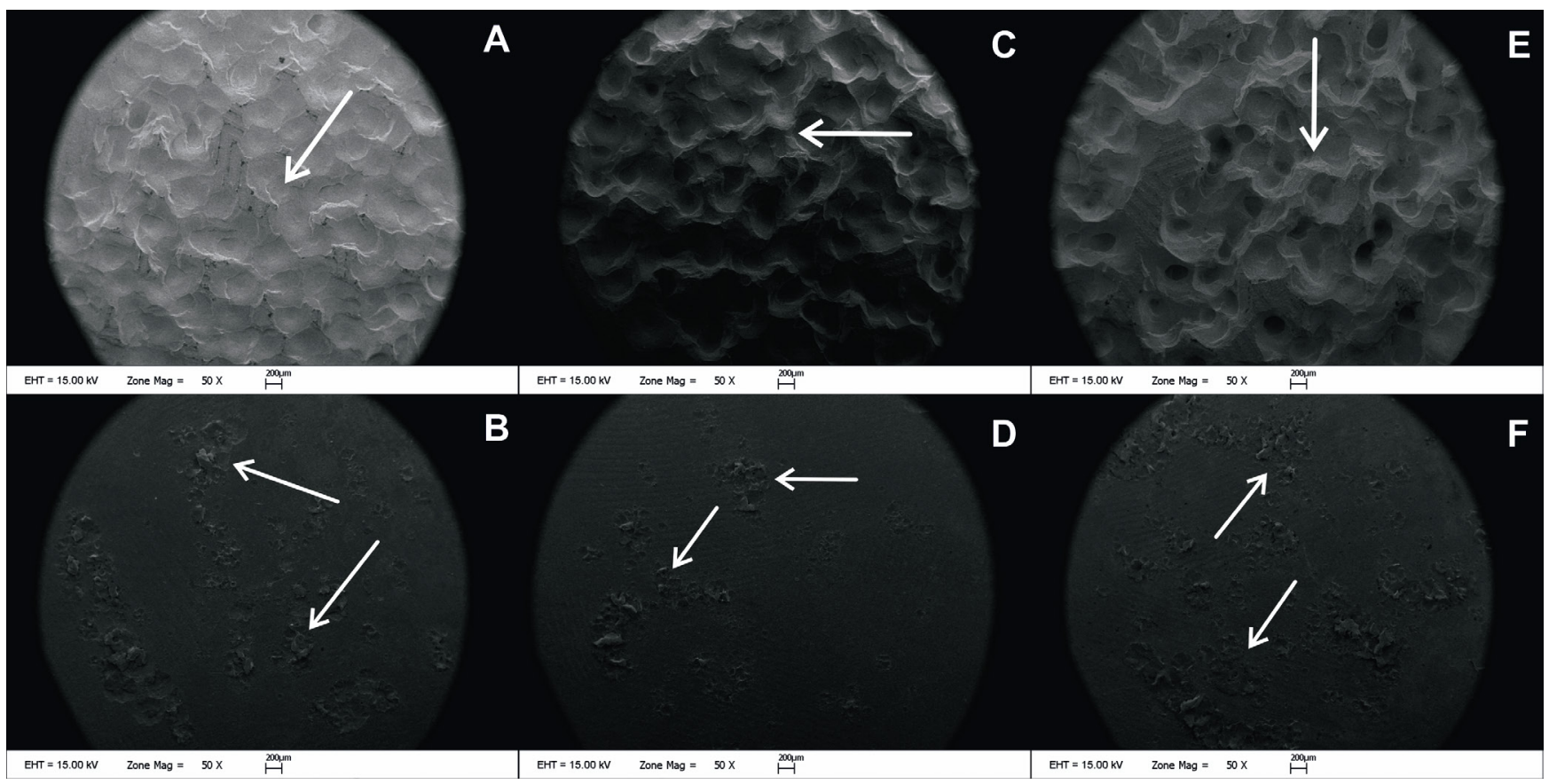

Fig. 6. Scanning electron microscopy (SEM) images of the pre-sintered zirconia surface after laser irradiation at $1 \mathrm{~W}(\mathrm{~A})$ and after sintering (B), of the presintered zirconia surface after laser irradiation at $2 \mathrm{~W}(\mathrm{C})$ and after sintering (D), and of the pre-sintered zirconia surface after laser irradiation at $3 \mathrm{~W}$ ( $\mathrm{E}$ ) and after sintering $(F)$

Arrows indicate melting areas; magnification $\times 500$.

morphology of the zirconia surface may consequently result in different SBS values. In the present study, it was found that the laser irradiation of the pre-sintered zirconia surface, which results in a chalk-like form that is not hard, formed a significantly greater retentive area (Fig. 6). This situation may have positively affected the SBS of zirconia to the resin cement. While the pre-sintered subgroups commonly presented a cohesive type of failure, other subgroups showed adhesive failure.

Although zirconia restorations are clinically successful, ${ }^{29}$ there are conflicting reports in the literature regarding the safest surface treatment to be used to enhance the lifespan of such restorations..$^{7,10,13,14,17}$ Having that in mind, the authors of this study believe that there is a need for improvement of the SBS between cements and the zirconia surface, while maintaining the integrity of the zirconia surface itself. Accordingly, the zirconia form and surface treatment to prepare the restoration for cementation are important factors to be taken into consideration.

\section{Limitations}

The $1^{\text {st }}$ limitation of the current study is that chemical bonds were not researched. A chemical bond, just as a mechanical bond, largely affects SBS. The chemical bond between the phosphate groups and zirconia has been previously suggested ${ }^{30}$ and might be the reason for different results in each group of the present study. Stronger adhesion between zirconia and the resin cement was obtained with the treatment of the pre-sintered zirconia surface. Restorations are exposed to many environmental factors in the oral cavity, such as moisture, and mechanical and thermal fatigue. The $2^{\text {nd }}$ limitation of this study is the absence of thermocycling. To simulate more realistic clinical conditions in in vitro studies, methods such as water storage, mechanical loading or thermocycling are used. Moreover, applying thermocycling or mechanical cycling in addition to thermocycling may be effective in obtaining more accurate results by simulating intraoral conditions. In addition to the effect of thermal cycles at $5^{\circ} \mathrm{C}$ and $55^{\circ} \mathrm{C}$, further studies could focus on the efficiency parameters of various lasers for future development. Also, it would be of interest to evaluate the surface topography of zirconia after different pretreatment by using confocal microscopy with SEM and AFM.

\section{Conclusions}

Within the limitations of this in vitro study, the following conclusions can be drawn:

- the surface treatment of pre-sintered zirconia with the 1-3 W Nd:YAG laser irradiation enhanced the SBS of pre-sintered zirconia to the resin cement;

- the airborne particle abrasion treatment in the presintered and sintered groups decreased the SBS of zirconia to the resin cement; and

- mechanical treatment generated stress on the zirconia surface and increased the $t-m$ phase transformation; the $\mathrm{m}-\mathrm{t}$ transformation occurs during the sintering process, when it is used after pre-sintering surface treatment. 
Although laser application is expensive, the fact that a single laser device has many application options (caries removal, restoration removal, canal irrigation, dentin sensitivity treatment, etc.) increases the demand for lasers. This situation makes researchers conduct in vitro studies on additional procedures that can be performed with lasers. In this context, this study showed the parameters and forms at which the laser can be applied to increase the adhesion of zirconia to a resin cement in addition to its routine application. Laser irradiation at the tested power levels may be preferred by clinicians on pre-sintered zirconia for improving SBS between a resin cement and presintered zirconia.

\section{ORCID iDs}

Türker Akar (1) https://orcid.org/0000-0003-2035-8686 Ayşe Dündar (1) https://orcid.org/0000-0001-6373-6267 Ömer Kırmalı (1) https://orcid.org/0000-0002-4313-344X Özlem Üstün (1) https://orcid.org/0000-0002-4756-1118 Alper Kapdan (1) https://orcid.org/0000-0001-5773-8522 Hakan Er (1) https://orcid.org/0000-0001-7739-4712 Alper Kuştarcı (1) https://orcid.org/0000-0002-4942-3739 Kürşat Er (10) https://orcid.org/0000-0002-0667-4909

Burak Yılmaz (1) https://orcid.org/0000-0002-7101-363X

\section{References}

1. Denry I, Kelly JR. State of the art of zirconia for dental applications. Dent Mater. 2008;24(3):299-307. doi:10.1016/j.dental.2007.05.007

2. Tinschert J, Schulze KA, Natt G, Latzke P, Heussen N, Spiekermann H. Clinical behavior of zirconia-based fixed partial dentures made of DC-Zirkon: 3-year results. Int J Prosthodont. 2008;21(3):217-222. PMID:18548959.

3. Hisbergues M, Vendeville S, Vendeville P. Zirconia: Established facts and perspectives for a biomaterial in dental implantology. JBiomed Mater Res B ApplBiomater. 2009;88(2):519-529. doi:10.1002/ jbm.b.31147

4. Hannink RHJ, Kelly PM, Muddle BC. Transformation toughening in zirconia-containing ceramics. J Am Ceram Soc. 2000;83(3):461-487. doi:10.1111/j.1151-2916.2000.tb01221.x

5. Kirmali O, Akin H, Ozdemir AK. Shear bond strength of veneering ceramic to zirconia core after different surface treatments. Photomed Laser Surg. 2013;31(6):261-268. doi:10.1089/pho.2013.3487

6. Kirmali O, Akin H, Kapdan A. Evaluation of the surface roughness of zirconia ceramics after different surface treatments. Acta Odontol Scand. 2014;72(6):432-439. doi:10.3109/00016357.2013.853320

7. Cavalcanti AN, Foxton RM, Watson TF, Oliveira MT, Giannini M, Marchi GM. Bond strength of resin cements to a zirconia ceramic with different surface treatments. Oper Dent. 2009;34(3):280-287. doi:10.2341/08-80

8. Akın H, Ozkurt Z, Kırmalı O, Kazazoglu E, Ozdemir AK. Shear bond strength of resin cement to zirconia ceramic after aluminum oxide sandblasting and various laser treatments. Photomed Laser Surg. 2011;29(12):797-802. doi:10.1089/pho.2011.3039

9. Liu D, Matinlinna JP, Tsoi JKH, et al. A new modified laser pretreatment for porcelain zirconia bonding. Dent Mater. 2013;29(5):559-565. doi:10.1016/j.dental.2013.03.002

10. Moon JE, Kim SH, Lee JB, Ha SR, Choi YS. The effect of preparation order on the crystal structure of yttria-stabilized tetragonal zirconia polycrystal and the shear bond strength of dental resin cements. Dent Mater. 2011;27(7):651-663. doi:10.1016/j.dental.2011.03.005

11. Zarone F, Sorrentino R, Vaccaro F, Traini T, Russo S, Ferrari M. Acid etching surface treatment of feldspathic, alumina and zirconia ceramics: A micromorphological SEM analysis. Int Dent South Afr. 2011;8(3):20-26. https://www.researchgate.net/publication/237303064_Acid_etching_surface_treatment_of_feldspathic_alumina_and_zirconia_ceramics_A_micromorphological_ SEM_analysis. Accessed on October 1, 2020.
12. Casucci A, Mazzitelli C, Monticelli F, et al. Morphological analysis of three zirconium oxide ceramics: Effect of surface treatments. Dent Mater. 2010;26(8):751-760. doi:10.1016/j.dental.2010.03.020

13. Kirmali O, Kapdan A, Kustarci A, Er K. Veneer ceramic to Y-TZP bonding: Comparison of different surface treatments. J Prosthodont. 2016;25(4):324-329. doi:10.1111/jopr.12304

14. Ghasemi A, Kermanshah H, Ghavam M, et al. Effect of Er,Cr:YSGG laser treatment on microshear bond strength of zirconia to resin cement before and after sintering. J Adhes Dent. 2014;16(4):377-382. doi:10.3290/j.jad.a32444

15. Gandolfi Paranhos MP, Burnett LH Jr., Magne P. Effect of Nd:YAG laser and $\mathrm{CO}_{2}$ laser treatment on the resin bond strength to zirconia ceramic. Quintessence Int. 2011;42(1):79-89. PMID:21206937.

16. Arami S, Tabatabae MH, Namdar SF, Chiniforush N. Effects of different lasers and particle abrasion on surface characteristics of zirconia ceramics. J Dent (Tehran). 2014;11(2):233-241. PMID:24910700. PMCID:PMC4043556.

17. Kirmali O, Kustarci A, Kapdan A, Er K. Efficacy of surface roughness and bond strength of Y-TZP zirconia after various pre-treatments. Photomed Laser Surg. 2015;33(1):15-21. doi:10.1089/pho.2014.3825

18. Liu L, Liu S, Song X, Zhu Q, Zhang W. Effect of Nd:YAG laser irradiation on surface properties and bond strength of zirconia ceramics. Lasers Med Sci. 2015;30(2):627-634. doi:10.1007/s10103-013-1381-7

19. Garvie RC, Nicholson PS. Phase analysis in zirconia systems. J Am Ceram Soc. 1972;55(6):303-305. doi:10.1111/j.1151-2916.1972.tb11290.x

20. Saka M, Yuzugullu B. Bond strength of veneer ceramic and zirconia cores with different surface modifications after microwave sintering. J Adv Prosthodont. 2013;5(4):485-493. doi:10.4047/jap.2013.5.4.485

21. Zanjani VA, Ahmadi $H$, Nateghifard A, et al. Effect of different laser surface treatment on microshear bond strength between zirconia ceramic and resin cement. J Investig Clin Dent. 2015;6(4):294-300. doi:10.1111/jicd.12105

22. Su N, Yue L, Liao $Y$, et al. The effect of various sandblasting conditions on surface changes of dental zirconia and shear bond strength between zirconia core and indirect composite resin. J Adv Prosthodont. 2015;7(3):214-223. doi:10.4047/jap.2015.7.3.214

23. Okutan Y, Kandemir B, Gundogdu Y, Kilic HS, Yucel MT. Combined application of femtosecond laser and air-abrasion protocols to monolithic zirconia at different sintering stages: Effects on surface roughness and resin bond strength. J Biomed Mater Res B Appl Biomater. 2021;109(4):596-605. doi:10.1002/jbm.b.34741

24. Henriques $B$, Fabris $D$, Souza JCM, et al. Bond strength enhancement of zirconia-porcelain interfaces via $\mathrm{Nd}$ :YAG laser surface structuring. J Mech Behav Biomed Mater. 2018;81:161-167. doi:10.1016/j.jmbbm.2018.02.031

25. Kirmali O, Kustarci A, Kapdan A. Surface roughness and morphologic changes of zirconia: Effect of different surface treatment. Niger J Clin Pract. 2015;18(1):124-129. doi:10.4103/1119-3077.146994

26. Akin GE, Akin H, Sipahi C, Piskin B, Kirmali O. Evaluation of surface roughness and bond strength of quartz fiber posts after various pre-treatments. Acta Odontol Scand. 2014;72(8):1010-1016. doi:10.3 109/00016357.2014.939710

27. Karthigeyan S, Ravindran AJ, Bhat RTR, Nageshwarao MN, Murugesan SV, Angamuthu V. Surface modification techniques for zirconia-based bioceramics: A review. J Pharm Bioallied Sci. 2019;11(Suppl 2):S131-S134. doi:10.4103/JPBS.JPBS_45_19

28. Usumez A, Hamdemirci N, Koroglu BY, Simsek I, Parlar O, Sari T. Bond strength of resin cement to zirconia ceramic with different surface treatments. Lasers Med Sci. 2013;28(1):259-266. doi:10.1007/ s10103-012-1136-x

29. Blatz MB, Vonderheide $M$, Conejo J. The effect of resin bonding on long-term success of high-strength ceramics. J Dent Res. 2018;97(2):132-139. doi:10.1177/0022034517729134

30. Wolfart M, Lehmann F, Wolfart S, Kern M. Durability of the resin bond strength to zirconia ceramic after using different surface conditioning methods. Dent Mater. 2007;23(1):45-50. doi:10.1016/j.dental.2005.11.040 